\title{
A robust free space optical communication system
}

\author{
Bosanta Ranjan Boruah \\ Department of Physics, Indian Institute of Technology Guwahati, Guwahati 781039, India. \\ Email: brboruah@iitg.ac.in
}

\begin{abstract}
Free space optical communication system sends information from one place to another optically through free space.One major issue of free space optical communication system remains tackling the effect of atmospheric turbulence in air through which the beam propagates. In this article we provide a brief overview of a recent development in which it is shown that using the property of orthogonal aberration modes, called Zernike modes, it is possible to send information from one place to another in a robust manner even in the presence of atmospheric turbulence.
\end{abstract}

Keywords: Free space optical communication (FSO); Zernike mode; atmospheric turbulence, wave front sensor.

\section{Introduction}

Free space optical communication (FSO) [Chan, V. W. , 2006] is a communication system to send information from one place to another through free space using the properties of a light beam which is mostly a laser beam. Figure 1 shows pictorial representation of a free space optical communication system, It primarily comprises a transmitter that encodes and transmits the user information by modulating the properties of a light beam and a receiver that receives the beam and decodes the user information.

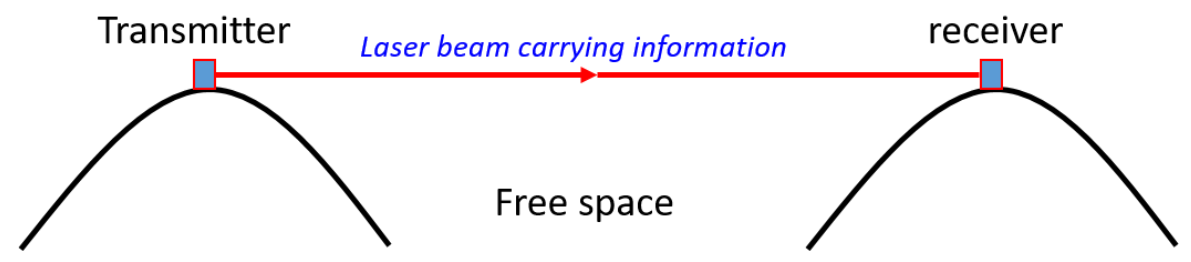

Figure 1: Transmission of informaiton from one place (i.e. the transmitter) to another (i.e. the receiver) through free space via a light beam. 
Such light based communication systems have several advantages, for instance, ability to send information with a very high data content. During last decade or so there has been a tremendous amount of developments in the area of free space optical communication especially using light beam carrying orbital angular momentum (OAM),after its feasibility was demonstrated [Gibson, G, 2004]. However OAM mode based free space communication systems suffer from a number of important limitations. Firstly the OAM modes do not form a complete basis set and consequently can not provide the optimal information content in the transmitted beam [Zhao, N., Li, X., Li, G. et al., 2015]. The OAM modes also suffer from the effect of atmospheric turbulence and the system does not have any inherent mechanism to compensate for the resulting inaccuracy in the transmitted data.

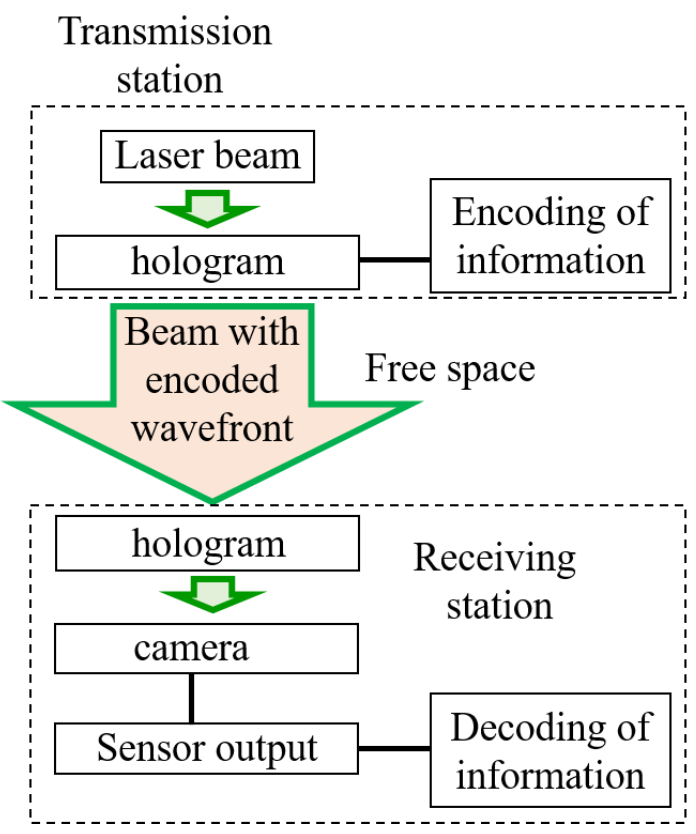

Figure 1 Block diagram depicting the Zernike mode based communication system

Fortunately the phase profile of a laser beam can also be modulated in terms of Zernike modes [Noll, R. J., 1976]. Zernike modes are, as mathematically defined, polynomials depending on the radial and azimuthal coordinates, with different modes orthogonal to one another and form a complete basis set. One may use computer generated holography [Boruah, B. R. 2009] to dynamically modulate the phase profile of a laser beam using one or more Zernike modes. The strengths of the Zernike modes present in an 
incident beam can be estimated in a single measurement using a type-K modal wavefront sensor [Konwar, S. \& Boruah, B. R, 2019]. The type-K sensor can provide a precise measure of the strength of each Zernike mode without getting affected by the presence or absence of other Zernike modes.

We have proposed a free space optical communication system where in the transmission station the wavefront of laser beam is modulated using a combination of Zernike modes whose strengths are mapped to the user data to be transmitted [Konwar, S., Boruah, B.R., 2020]. A block diagram of the system is shown in the figure 2 . The laser beam carrying the user information in its phase profile travels through the free space before it arrives at the receiving station. At the receiving station, the beam is incident on a type-K modal wavefront sensor comprising a type-K hologram and a camera. The output of the type-K sensor is then used to obtain the user information.

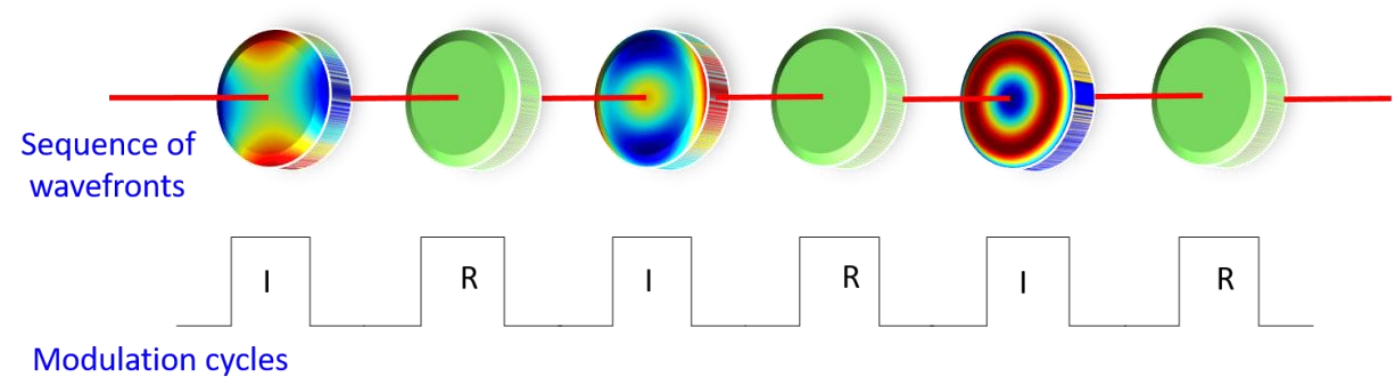

Figure 3 Sequence of information carryimg wavefronts (I) followed by reference wavefront (R) in alternate modulation cycles to compensate for atmospheric turbulence

\subsection{Full-wave solution}

In order to compensate for the effect of turbulence the scheme sends each information carrying wavefront immediately followed by a reference wavefront as shown in figure 3 . The reference wavefront is used to quantify the influence of turbulence in terms of Zernike modes using the property that Zernike modes form a complete basis set. The type-K sensor output for the reference wavefront is then used to make correction in the receiver signal for the preceding information carrying wavefront.

We have showed experimentally that our aberration compensation scheme effectively removes in inaccuracy in the transmitted data due to turbulence. We have also showed that the information content of the beam per modulation cycle using two different Zernike modes in our scheme can be 106 time more that the same for an FSO using two different OAM modes. 


\section{Reference}

Chan, V. W. Free-space optical communications. J. Lightwave Technol.24, 4750-4762 (2006)..

Gibson, G. et al. Free-space information transfer using light beams carrying orbital angular momentum. Opt. Express12, 5448-5456 (2004).

Zhao, N., Li, X., Li, G. et al. Capacity limits of spatially multiplexed free-space communication. Nature Photon9, 822-826 (2015).

Noll, R. J. Zernike polynomials and atmospheric turbulence. JOSA 66, 207-211 (1976)

Boruah, B. R. Dynamic manipulation of a laser beam using a liquid crystal spatial light modulator. Am. J. Phys. 77, 331-336 (2009)

Konwar, S. \& Boruah, B. R. Improved linear response in a modal wavefront sensor. J. Opt. Soc. Am. A36, 741-750 (2019).

Konwar, S., Boruah, B.R. Leveraging the orthogonality of Zernike modes for robust free-space optical communication. Commun Phys3, 203 (2020). 